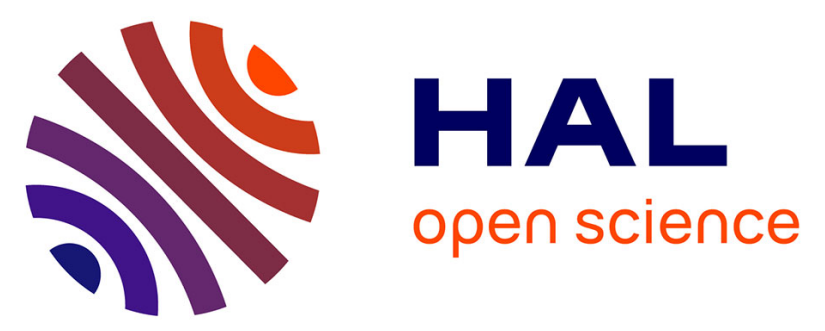

\title{
Real-Time Look-Ahead Distance Optimization for Smooth and Robust Steering Control of Autonomous Vehicles
}

Hussam Atoui, V Milanés, Olivier Sename, John Jairo Martinez Molina

\section{- To cite this version:}

Hussam Atoui, V Milanés, Olivier Sename, John Jairo Martinez Molina. Real-Time Look-Ahead Distance Optimization for Smooth and Robust Steering Control of Autonomous Vehicles. MED 2021 - 29th Mediterranean Conference on Control and Automation, Jun 2021, Bari (virtual), Italy. 10.1109/MED51440.2021.9480235 . hal-03301885

HAL Id: hal-03301885

https://hal.univ-grenoble-alpes.fr/hal-03301885

Submitted on 27 Jul 2021

HAL is a multi-disciplinary open access archive for the deposit and dissemination of scientific research documents, whether they are published or not. The documents may come from teaching and research institutions in France or abroad, or from public or private research centers.
L'archive ouverte pluridisciplinaire HAL, est destinée au dépôt et à la diffusion de documents scientifiques de niveau recherche, publiés ou non, émanant des établissements d'enseignement et de recherche français ou étrangers, des laboratoires publics ou privés. 


\title{
Real-Time Look-Ahead Distance Optimization for Smooth and Robust Steering Control of Autonomous Vehicles*
}

\author{
H. Atoui ${ }^{1,2}$, V. Milanés ${ }^{1}$, O. Sename ${ }^{2}$ and John J. Martinez ${ }^{2}$
}

\begin{abstract}
This paper presents an optimization problem for the look-ahead distance used in lateral automated vehicle control. A relation is formulated to describe the sensitivity of the control input steering speed $\dot{\delta}$ with respect to the variation of the look-ahead distance $L$. The nonlinear relation is written as an LPV model using the grid-based approach. The aim is to design a controller which minimizes the steering speed $\dot{\delta}$ by optimizing an additional look-ahead distance $L_{d}$ that is added to the nominal look-ahead distance. Such action generates a smooth/stable vehicle motion when subjected to high oscillations due to noises, large lateral errors, etc. The proposed solution is based on the Linear Parameter Varying (LPV) control approach, where an output-feedback dynamic controller is designed based on Linear Matrix Inequalities (LMIs). The control synthesis is carried out using the grid-based approach combined with the $\mathcal{H}_{\infty}$ control problem. Simulation results show the tracking performance and the smoothness of the steering input, when the vehicle is subjected to successive large lateral errors, which provides a comfortable riding.
\end{abstract}

\section{INTRODUCTION}

Day by day, automated vehicles are entering a new level of intelligence. The lateral vehicle control is vital problem to achieve an on-road automated movement. It is important as much as it is critical where it concerns lane-keeping, lanechanging, obstacle avoidance, autonomous parking, etc. Most of the path-tracking methods in practice encounter challenges when high accuracy and robustness are required [1]. The first role of the lateral control is to keep the vehicle within the boundaries of a lane, which is performed by adjusting the steering actuator to minimize the lateral error between the vehicle position and a target point in the generated reference. The question comes on how to choose the target point. If the target point is chosen to be the closest point (in the reference) to the vehicle, the actuator may not be able to minimize the corresponding lateral error in case of high speeds, high curvature changes, etc. This is due to delays found in the used sensors and actuators. Then, a look-ahead approach is raised to predict the future lateral error at a chosen lookahead distance. Look-ahead systems use sensors as machine vision, radar, and LiDAR, to measure the lateral displacement in front of the vehicle.

*Research Department, Renault SAS, 1 Avenue de Golf, Guyancourt 78280, France

1 H. Atoui and V. Milanés are with the Research Department, Renault SAS, 1 Avenue de Golf, 78280 Guyancourt, France hussam.atoui, vicente.milaneserenault. com

${ }^{2}$ H. Atoui, O. Sename and John J. Martinez are with GIPSAlab, CNRS, Univ. Grenoble Alpes, Grenoble INP, 38000 Grenoble, France. hussam. Atouidetu.univ-grenoble-alpes.fr, olivier.senamedgrenoble-inp.fr,

john-jairo.martinez-molina@grenoble-inp. fr
In 90s, the control concepts based on look-ahead systems have been raised. These concepts have been developed to mainly improve the efficiency and performance of the longitudinal and lateral vehicle controls. [2] proposes a new approach to improve the vehicle trajectory prediction for the Adaptive Cruise Control (ACC) system. It concludes that the more look-ahead distance increases, the greater the prediction errors. Moreover, the look-ahead approach is also used to optimize traffic flow, minimize trip time [3], decrease fuel consumption and provide safety for heavy-duty vehicles [4], [5], [6].

On the other hand, look-ahead systems are used for lateral objectives as shown in the pioneering works [7], [8]. In [9] and [10], the authors have recognized that the closed-loop stability is sensitive to the variation of speed. Specifically, when the vehicle speed increases, the closed-loop zeros and poles move toward the imaginary axis leading to poor damping of the poles. Increasing the look-ahead distance moves the zeros closer to the real axis, improving the damping of the closed-loop poles. As a result, the choice of a proper look-ahead distance is important for stability and performance of the closed-loop system. Practically, as much as the look-ahead distance decreases, the vehicle will lose farer information which leads to periodic oscillations due to actuators and sensors delays. On the other hand, when the look-ahead distance increases, the vehicle may not be able to deal with near obstacles or maneuvers. A relation has been analyzed between the look-ahead distance and the longitudinal velocity, the road curvature and the processing delay of the vision. In [11], the authors introduce an equation which calculates the look-ahead distance as a function of vehicle specifications and longitudinal speed taking into account the distance between the vehicle and a bumper. [12] proposes a dynamic look-ahead distance $L(s)$ which varies with respect to speed. The aim was to obtain the lateral acceleration independent of the longitudinal speed which makes the design of the steering control much easier.

Recently, several studies aim to find the best tuning of the look-ahead distance $L$ with respect to the vehicle speed $v_{x}$. The studies in [13], [14] and [15] propose to tune $L$ from numerical analysis. [16] estimates $L$ manually by analyzing the closed-loop poles with respect to speed, lookahead distance, and lateral control feedback gains. In [17], the authors indicate three look-ahead distances $\left(L_{1}, L_{2}\right.$, $\left.L_{3}\right)$ at a fixed speed. Then, a feedback lateral control uses vehicle lateral deviations at the indicated look-ahead points to improve the performance of the vehicle at different road curvatures. In [18], the look-ahead distance is formulated 


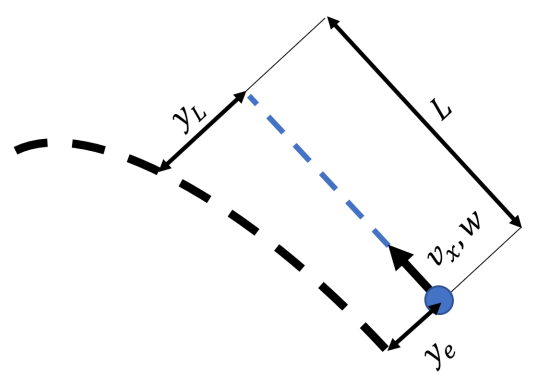

Fig. 1: Lateral control based on look-ahead concept

as a linear function of speed by choosing a suitable lookahead time as a slope, with lower and upper bounds. The same methodology is also introduced in [19] and [20]. [21] and [22] propose a simplified adaptive method that tunes the look-ahead distance from the commanded speed instead of the measured one to improve a path prediction process. The tuning method parameters are obtained from a number of different experiences. Other works employ the fuzzy logic approach to tune the look-ahead distance [23], [24]. They consider the road curvature and the current vehicle lateral error for the selection of the look-ahead distance.

As an overall, the look-ahead distance may be determined based on at least one of the following: vehicle speed, rotating speed, steering acceleration, steering angle, heading angle, lane curvature, current lateral error, predicted lateral error, distance to lane boundary, vehicle rotating performance, obstacle location. Tuning the look-ahead distance based only on the vehicle speed is not sufficient, since it could face some critical situations as large lateral errors for example. This paper proposes a method to find, in real-time, an optimal look-ahead distance according to the vehicle speed and steering acceleration, and taking into consideration the vehicle heading error. This improves the vehicle performance when subjected to high noises at highways, to large lateral errors that may occur at the autonomous mode initialization or sudden lane-changes, etc.

The paper is organized as follows: Section 2 defines the lateral control based on the concept of look-ahead distance. The invented look-ahead system is introduced in Section 3. Section 4 compares the vehicle performance with and without using the invented look-ahead system. Finally, some concluding remarks are given in Section 5.

\section{LATERAL CONTROL BASED ON LOOK-AHEAD DISTANCE}

The lateral control scheme usually contains two main components [16],[25]: 1) A feedforward term which concerns the reference trajectory by considering the road curvature and vehicle speed (see Fig. 1). The road curvature is estimated at a target point chosen in front by a look-ahead distance in order to predict the future lateral displacement; 2) A feedback compensator which activates the steering actuator to minimize the current vehicle errors and correct its lateral and heading positions.

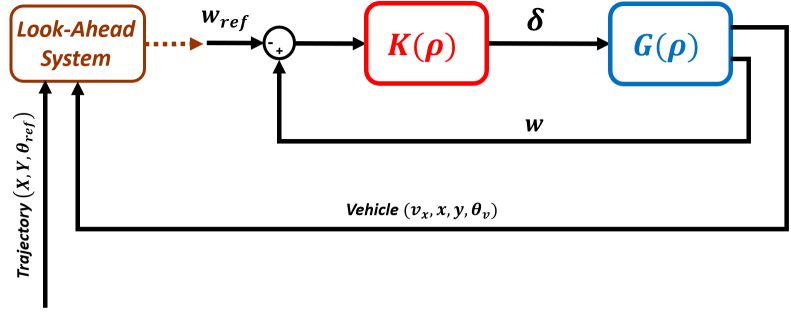

Fig. 2: Lateral control scheme

Referring to [26] and [27], the lateral dynamic model is derived as:

$$
\left\{\begin{array}{l}
\dot{v}_{y}=\frac{F_{y f} \cos \delta+F_{y r}}{m}-w v_{x} \\
\dot{w}=\frac{F_{y f} l_{f} \cos \delta-F_{y r} l_{r}}{I}
\end{array}\right.
$$

where $v_{x}, v_{y}$ and $w$ are the longitudinal, lateral and rotational velocities in the vehicle's frame, respectively. $\delta$ is the control input, the steering angle of the front tire. $F_{y f}$ and $F_{y r}$ are the lateral forces applied to the front and rear tires, respectively. $I, m, l_{f}$ and $l_{r}$ are the vehicle's inertia, mass and the distance from the center of gravity to the front and rear wheel axes respectively. The lateral forces are approximated to be:

$$
\begin{gathered}
F_{y f}=C_{f}\left(\delta-\frac{v_{y}}{v_{x}}-\frac{l_{f} w}{v_{x}}\right), \\
F_{y r}=C_{r}\left(-\frac{v_{y}}{v_{x}}+\frac{l_{r} w}{v_{x}}\right),
\end{gathered}
$$

where $C_{f}$ and $C_{r}$ represent the stiffness of the front and rear wheel-tires.

Fig. 2 shows the scheme of the lateral control. $G(\rho)$ is the parameter-varying model describing the vehicle lateral dynamics (taking $\cos (\delta) \approx 1$ ) with $\rho=v_{x}$ as:

$$
G(\rho)\left\{\begin{array}{l}
\dot{x}(t)=A(\rho) x(t)+B u(t) \\
y(t)=C x(t)
\end{array}\right.
$$

where:

$$
\begin{aligned}
& x(t)=\left[\begin{array}{l}
v_{y} \\
w
\end{array}\right], u(t)=\delta, B=\left[\begin{array}{c}
\frac{1}{m} C_{f} \\
\frac{1}{I} C_{f} l_{f}
\end{array}\right], C=\left[\begin{array}{ll}
0 & 1
\end{array}\right], \\
& A(\rho)=\left[\begin{array}{cc}
-\frac{C_{r}+C_{f}}{m v_{x}} & -\frac{C_{f} l_{f}-C_{r} l_{r}}{m v_{r}}-v_{x} \\
-\frac{C_{f} l_{f}-l_{r} C_{r}}{I v_{x}} & -\frac{C_{f} l_{f}^{2}+l_{r}^{2} C_{r}}{I v_{x}}
\end{array}\right],
\end{aligned}
$$

The longitudinal speed to be bounded as:

$$
v_{x} \in\left[v_{x_{\text {min }}}, v_{x_{\text {max }}}\right] \mathrm{m} / \mathrm{s}
$$

Fig. 2, $K(\rho)$ represents a parameter-varying lateral control designed using any control concept (pole-placement, PID, $\mathcal{H}_{\infty}, \ldots$... . Notice that this controller can be designed using any of the LPV control approaches; polytopic, grid-based, or LFT approach (see [28]). This paper uses the grid-based LPV control approach to design an LPV/H $\mathcal{H}_{\infty}$ output feedback controller $K(\rho)$ [29]. The aim is to track the yaw rate reference $w_{\text {ref }}$ and respecting the actuator limitations. A grid-based LPV system is a set of LTI systems linearized at different operating points. It is usually chosen due to its low conservatism compared to the other approaches. 
The Look-Ahead System (LAS) block presented in Fig. 2 aims to generate a coherent yaw-rate reference $w_{\text {ref }}$. The LAS uses the current vehicle situation measured by the sensors and the information from the navigation system. The main role of LAS is to improve the lane-tracking accuracy and driving comfort at the same time. From [16], it is shown that $w_{\text {ref }}$ can be approximated as:

$$
w_{\text {ref }}=\frac{2 v_{x} y_{L}}{L^{2}}
$$

where $y_{L}$ is the predicted lateral error at the look-ahead distance $L$ (see Fig. 1). The way to find an optimal value of $L$ is the objective of this paper.

\section{AdVAnCED LoOK-AHEAd System}

The role of LAS has been often concerned to adapt the look-ahead distance $L$ with respect to the longitudinal speed $v_{x}$. Although it has provided good performance at normal cases (small lateral errors, low steering noises,...), however, it cannot provide the required performance level for all situations that often face an autonomous vehicle. Indeed, such situations are found when the vehicle starts its autonomous mode with an initial large lateral error, performs sudden lanechanges to avoid obstacles, and when the steering actuator is subjected to high noises/oscillations at high speeds, etc.

This work aims to propose an optimal LAS by considering the steering speed $\dot{\delta}$ and the heading error $\theta_{e}$ in addition to $v_{x}$ as parameters of the look-ahead distance optimization. The new look-ahead distance $\left.L_{(} v_{x}, \dot{\delta}, \theta_{e}\right)$ is here computed as:

$$
L\left(v_{x}, \dot{\delta}, \theta_{e}\right)=L_{v}\left(v_{x}\right)+L_{d}\left(\dot{\delta}, \theta_{e}\right)
$$

where $L_{v}$ is given from the look-up table which is tuned in terms of $v_{x}$ as done in the literature. $L_{d}$ represents a corrective term added to $L_{v}$ when the vehicle faces high steering speeds $\dot{\delta}$, then, the objective of $L_{d}$ is to smooth the generated $w_{\text {ref }}$. On the other hand, if $\theta_{e}$ is larger than a pre-defined threshold $T_{\theta}$ (i.e. $\theta_{e}>T_{\theta}$ ), the effect of $L_{d}$ is decreased to provide a faster heading error minimization. In this study, $L_{d}$ is computed using a controller which minimizes $\dot{\delta}$. To do so, a dynamic model is proposed as the sensitivity of the steering speed $\dot{\delta}$ with respect to the lookahead distance. The next section shows the model derivation steps.

\section{A. Model Formulation}

According to Fig. 2, a state-space Single-Input-SingleOutput (SISO) system between the control input $\delta$ and the reference trajectory $w_{\text {ref }}$ can be derived as:

$$
\delta(t)=[1+G(\rho) K(\rho)]^{-1} K(\rho) w_{r e f}(t)
$$

where $\rho=v_{x}$. At each grid point of $\rho$, let us transform the SISO system in (8) to a transfer function in Laplace domain, and multiply it by the complex variable $s$. Then, after performing an inverse Laplace transformation, and using (6), the steering speed $\dot{\delta}$ can be written in state-space as:

$$
\begin{aligned}
\dot{\delta}(t) & =\Gamma(\rho) w_{r e f}(t) \\
& =\Gamma(\rho) \frac{2 v_{x}(t) y_{L}(t)}{L_{d}^{2}(t)}
\end{aligned}
$$

where $\Gamma(\rho)$ represents the inverse Laplace transform of $\frac{s K(s)}{1+G(s) K(s)}$ (that must be proper), here its statespace matrices are $\left\{A_{\Gamma}(\rho), B_{\Gamma}(\rho), C_{\Gamma}(\rho), D_{\Gamma}(\rho)\right\}$. After reformulation, the steering speed $\dot{\delta}$ can be written as:

$$
\begin{aligned}
\dot{\delta}(t) & =\Gamma(\rho) \frac{2 v_{x}(t) y_{L}(t)}{L_{d}^{3}(t)} L_{d}(t) \\
& =\Sigma(\theta) L_{d}(t)
\end{aligned}
$$

where $\Sigma(\theta)=\Gamma(\rho) \frac{2 v_{x} y_{L}}{L_{d}^{3}}$ is an LPV model that varies with respect to $\theta=\left[v_{x}, L_{d}, y_{L}\right]^{T}$, and its corresponding statespace representation is written as:

$$
\Sigma(\theta)\left\{\begin{array}{l}
\dot{x}_{\Sigma}(t)=A_{\Sigma}(\theta) x_{\Sigma}(t)+B_{\Sigma}(\theta) L_{d}(t) \\
\dot{\delta}(t)=C_{\Sigma}(\theta) x_{\Sigma}(t)+D_{\Sigma}(\theta) L_{d}(t)
\end{array}\right.
$$

where $A_{\Sigma}(\theta)=A_{\Gamma}(\rho), C_{\Sigma}(\theta)=C_{\Gamma}(\rho), B_{\Sigma}(\theta)=$ $B_{\Gamma}(\rho) \frac{2 v_{x} y_{L}}{L_{d}^{3}}$, and $D_{\Sigma}(\theta)=D_{\Gamma}(\rho) \frac{2 v_{x} y_{L}}{L_{d}^{3}}$. It is worth mentioning that $\Sigma(\theta)$ is written as a grid-based LPV model where $v_{x}$ has the same gridded axis as in $K(\rho), L_{d}$ and $y_{L}$ are gridded on different axes and bounded in $[0,20] \mathrm{m}$ and $[0.1,4] m$ respectively.

The grid-based LPV approach can use any kind of interpolation (linear or nonlinear) between the gridded models to compute the LPV model. Suppose that, at an instant,

$$
\left[\begin{array}{l}
v_{x} \\
L_{d} \\
y_{L}
\end{array}\right] \in\left[\begin{array}{c}
v_{x, i}, v_{x, i+1} \\
L_{d, j}, L_{d, j+1} \\
y_{L, k}, y_{L, k+1}
\end{array}\right]
$$

the linear interpolation of the state-space matrices is done in a cubic region defined by the boundaries of the three parameters as:

$$
\left[\begin{array}{c|c}
A_{\Sigma}(\theta) & B_{\Sigma}(\theta) \\
\hline C_{\Sigma}(\rho) & D_{\Sigma}(\theta)
\end{array}\right]=\sum_{m=1}^{2^{3}} \alpha_{m}(\theta)\left[\begin{array}{c|c}
A_{m} & B_{m} \\
\hline C_{m} & D_{m}
\end{array}\right],
$$

where $\alpha_{m}(\theta) \in \mathbb{R}$ are the interpolating coefficients such that $\sum_{m=1}^{2^{3}} \alpha_{m}(\theta)=1$. The following section introduces the control design which aims to find an optimal value of $L_{d}$ to minimize the steering speed $\dot{\delta}$.

\section{B. Control Design}

Fig 3 depicts the control block diagram. $K_{L}(\theta)$ represents the LPV controller to be designed using gridding approach [29] achieving some required performances.

The $\mathcal{H}_{\infty}$ concept is chosen to minimize robustly the steering speed $\dot{\delta}$ which is subjected to high noises. Control performance requirements in $\mathcal{H}_{\infty}$ control theory are given by frequency domain functions. Two weighting functions $W_{e}$ and $W_{u}$ are used to achieve $\dot{\delta}$ minimization and $L_{d}$ limitation performances respectively. The objective is to achieve both performances with a trade-off between minimizing the lateral acceleration (caused by $\dot{\delta}$ ) and limiting the look-ahead distance rate. 


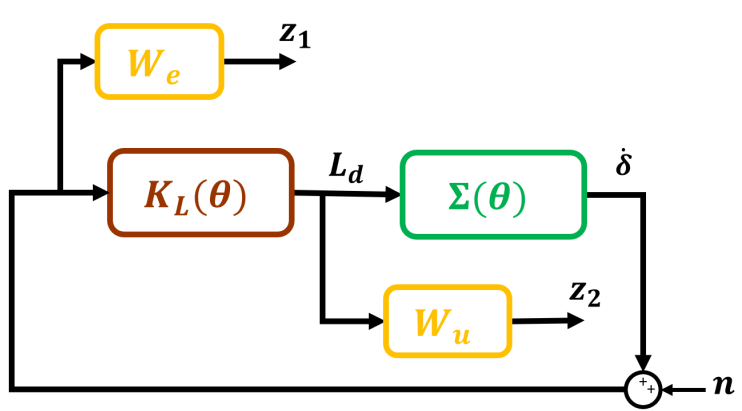

Fig. 3: Control design scheme

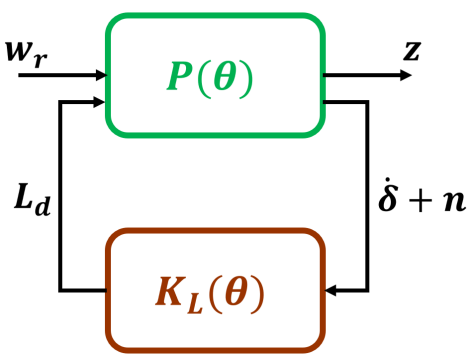

Fig. 4: General control configuration

\section{Tracking specification $\left(W_{e}\right)$}

The weighting transfer function is designed as:

$$
W_{e}(s)=\frac{\frac{s}{M_{s}}+w_{b}}{s+w_{b} \epsilon}
$$

where the parameters $M_{s}, w_{b}$ and $\epsilon$ are tuned as follows:

- $M_{s}=2$, to ensure robustness at any frequency.

- $w_{b} \geq 10$, to choose the rate of minimization.

- $\epsilon \leq 10^{-1}$, to represent the steady-state tracking error.

\section{Specification on the control input limitations $\left(W_{u}\right)$}

A filter is used to restrict the look-ahead distance control $L$. This filter is tuned as:

$$
W_{u}(s)=\frac{s+\frac{w_{b_{u}}}{M_{u}}}{\epsilon_{u} s+w_{b_{u}}}
$$

The parameters $M_{u}, w_{b_{u}}$ and $\epsilon_{u}$ are adopted as:

- $M_{u}$ represents the limitations on the maximum allowed $L_{d}$

- $w_{b_{u}}$, is related to the bandwidth $L_{d}$.

- $\epsilon_{u} \leq 10^{-2}$, is concerned with the noise rejection from $L_{d}$ at high frequencies.

\section{E. Generalized Plant}

Using the $\Sigma(\theta)$ and the weighting functions $W_{e}$ and $W_{u}$, Fig. 3 is transformed to build a general control configuration as in Fig. 4. The generalized plant $P(\theta)$ includes $\Sigma(\theta)$ in addition to the chosen weights. Thus the state vector of $P(\theta)$ is $x_{P}=\left[\begin{array}{lll}x_{\Sigma} & x_{W_{e}} & x_{W_{u}}\end{array}\right]^{T}$, and the controlled output $z=$ $\left[\begin{array}{ll}z_{1} & z_{2}\end{array}\right]^{T}$ represents the objective function to be optimized. $w_{r}=\left[\begin{array}{lll}r & d & n\end{array}\right]^{T}$ is the exogenous input, where $r, d$ and $n$ are the desired reference, input disturbance and the sensor

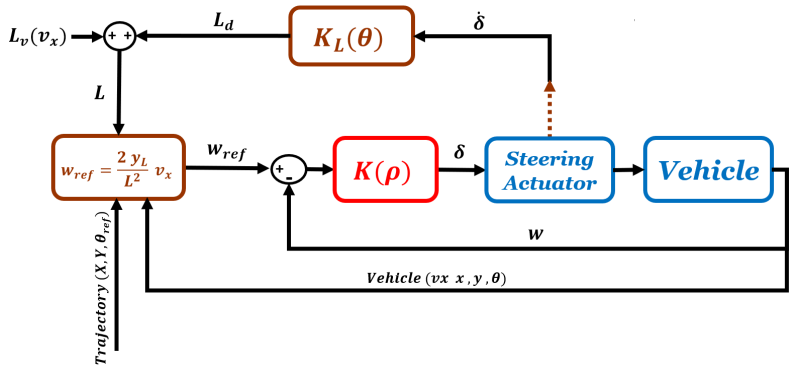

Fig. 5: Implementation scheme

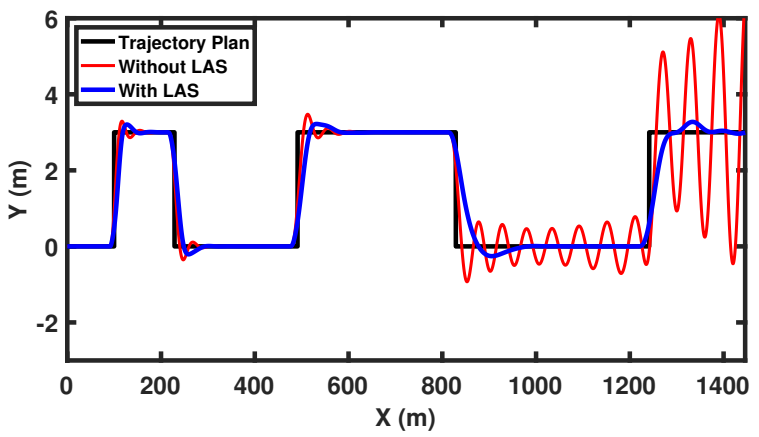

Fig. 6: Planned and controlled trajectories

noises respectively. The state-space representation of $P(\theta)$ (see Fig. 4) has the form:

$$
\left[\begin{array}{c}
\dot{x}_{P} \\
z \\
\dot{\delta}+n
\end{array}\right]=\left[\begin{array}{c|cc}
A_{P}(\theta) & B_{1}(\theta) & B_{2}(\theta) \\
\hline C_{1}(\theta) & D_{11}(\theta) & D_{12}(\theta) \\
C_{2}(\theta) & D_{21}(\theta) & D_{22}(\theta)
\end{array}\right]\left[\begin{array}{c}
x_{P} \\
w_{r} \\
u
\end{array}\right]
$$

Here, the varying parameters are chosen to be bounded as follows: $v_{x} \in[3,30] \mathrm{m} / \mathrm{s}, L_{d} \in[0,20] \mathrm{m}$, and $y_{e} \in$ $[0.1,4] \mathrm{m}$. The grid-based approach formulates the problem in the context of robust stability [30] by using a parameterdependent Lyapunov function along the gridded axes. The parameter-varying Lyapunov function $X(\theta)$ is chosen to be linearly dependent (order 1) on $\theta$ :

$$
X(\theta)=X_{0}+\theta X_{1},
$$

where $X_{0}$ and $X_{1}$ are unknown constant matrices to be computed from the Linear Matrix Inequalities (LMIs) shown in [29]. As a result, a set of LTI controllers is obtained where each one corresponds to a frozen value in the gridded space of the varying parameters. The controller $K(\theta)$ can be linearly interpolated as:

$$
\left[\begin{array}{c|c}
A_{k}(\theta) & B_{k}(\theta) \\
\hline C_{k}(\theta) & D_{k}(\theta)
\end{array}\right]=\sum_{m=1}^{2^{3}} \alpha_{m}(\theta)\left[\begin{array}{c|c}
A_{k, m} & B_{k, m} \\
\hline C_{k, m} & D_{k, m}
\end{array}\right],
$$

where $\alpha_{m}(\theta) \in \mathbb{R}$ are the interpolating coefficients such that $\sum_{m=1}^{2^{3}} \alpha_{m}(\theta)=1$.

\section{SIMULATION RESULTS}

The proposed Look-Ahead System (LAS) is implemented according to Fig. 5. The simulations are implemented on a 


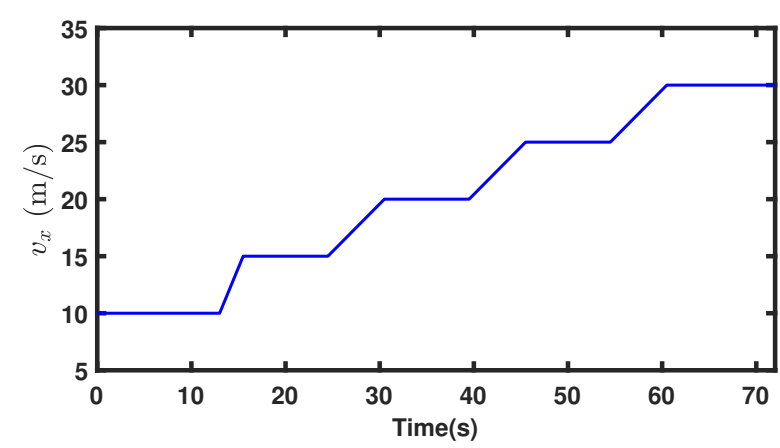

Fig. 7: Longitudinal speed $v_{x}(\mathrm{~m} / \mathrm{s})$

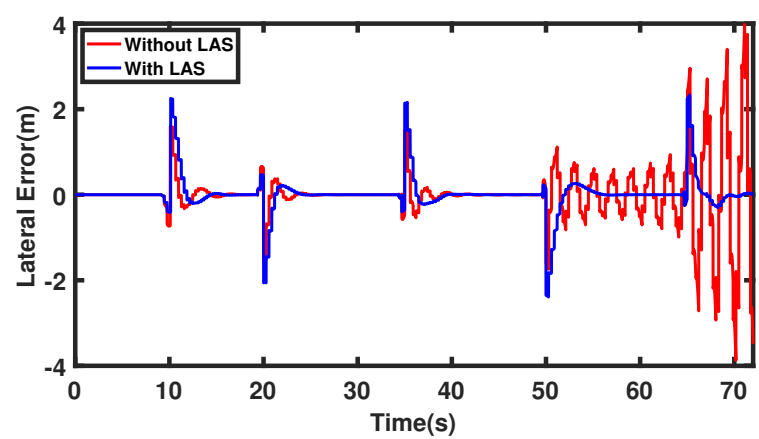

Fig. 8: Lateral error (m)

nonlinear model of a real Renault ZOE vehicle in discretetime domain with a sampling time $T_{s}=10 \mathrm{~ms}$. The nonlinear model includes nonlinear tire dynamics and estimated aerodynamic friction. To evaluate the efficiency of the developed Look-Ahead System (LAS), two tests are done: 1) without activating LAS $\left(L=L_{v}\right)$; and 2) activating LAS $\left(L=L_{v}+L_{d}\right)$. Recall that if $\theta_{e}$ is larger than a pre-defined threshold $T_{\theta}$ (i.e. $\theta_{e}>T_{\theta}$ ), the effect of $L_{d}$ is decreased to provide a faster heading error minimization. A scenario is chosen with five successive sudden lane changes with different longitudinal speeds. Fig. 6 depicts the planned and the controlled trajectories. The longitudinal speed profile is shown in Fig. 7 and the resultant lateral error in presented in Fig. 8.

Fig. 9 shows two sub-figures corresponding to the generated yaw rate references $w_{\text {ref }}$ in case of activating the LAS (Fig. 9b) and without its activation (Fig. 9a). It is worth to mention how the LAS affects the evolution of $w_{\text {ref }}$, where it is adapted suddenly when reaching high values thanks to the fast adaptation of the look-ahead distance $L$ (see Fig. 10). Additionally, the lateral controller $K(\rho)$ couldn't work on such high frequency which prevents it from aggressive tracking (check when time $\in[30,40] s$ in Fig. 9). Fig. 10 presents the variation of the nominal look-ahead distance (in red) accordingly with the evolution of speed, and the adaptive look-ahead distance (in blue) accordingly with the speed and the steering speed together.

Fig. 11 and 12 depicts the steering effort for both cases with/without activating the LAS. It can be shown that the

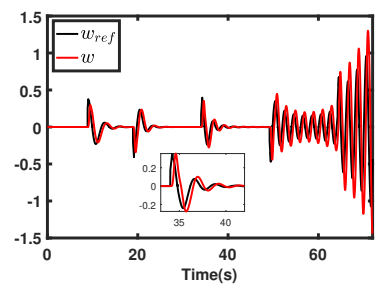

(a) without LAS

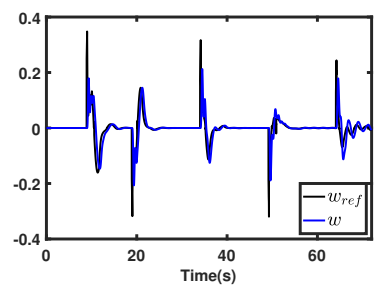

(b) with LAS
Fig. 9: Yaw rate reference $w_{r e f}$ and output $w(\mathrm{rad} / \mathrm{s})$

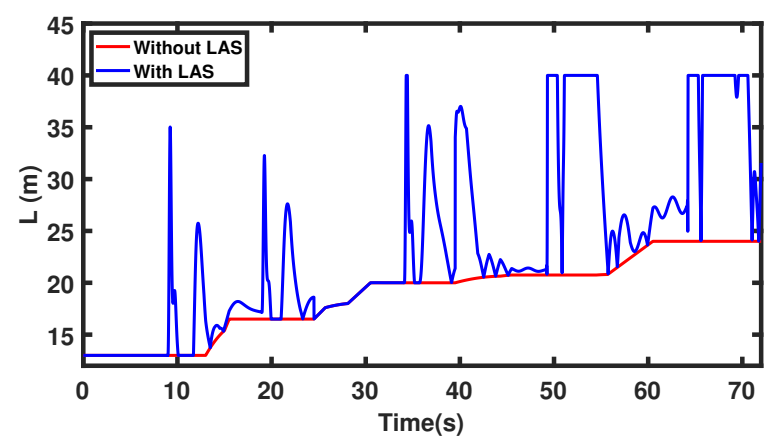

Fig. 10: Look-ahead distance $L(\mathrm{~m})$

LAS optimizes the steering effort (see Fig. 11). Moreover, Fig. 12 presents the advantage of the LAS in providing smooth transitions of the steering speed $\dot{\delta}$, which provides better driving comfort. Overall, Fig. 8, 11, and 12 show that the tracking and steering performances (when activating the LAS) are almost similar for all the speed range, which clarify the advantage and importance of the proposed LAS.

\section{CONCLUSION}

This paper has proposed an adaptive Look-Ahead System (LAS) to generate an optimal reference for the lateral control of autonomous vehicle. A grid-based LPV/H $\mathcal{H}_{\infty}$ controller is designed to minimize the steering speed $\dot{\delta}$ by finding an optimal look-ahead distance $L$. The results obtained in simulation shows the advantage of the LAS in improving the driving comfort and avoid lateral oscillations in critical situations. This system will be tested on a real RENAULT vehicle in the future to improve an the performance of an

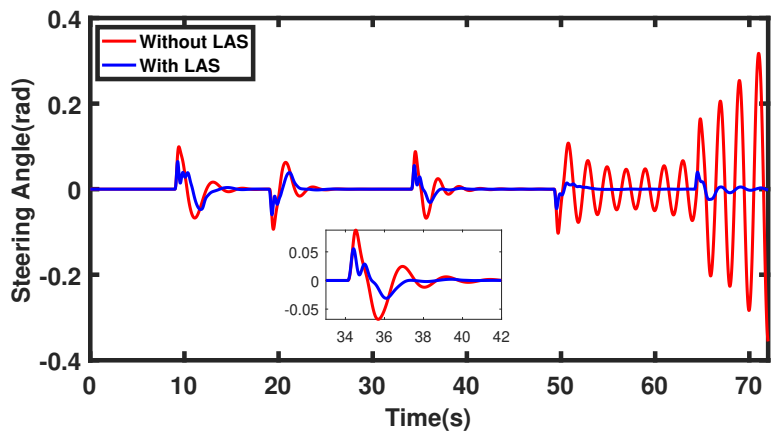

Fig. 11: Steering angle $\delta$ (rad) 


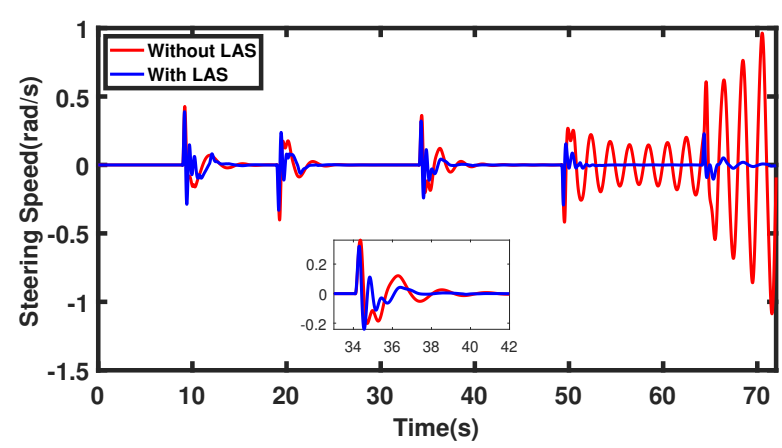

Fig. 12: Steering speed $\dot{\delta}(\mathrm{rad} / \mathrm{s})$

existing lateral controller. It provides better tracking accuracy simultaneously with actuator effort optimization in various experimental conditions.

\section{ACKNOWLEDGMENT}

Authors express their gratitude to RENAULT research department for its support in developing simulations on a nonlinear model of a real Renault ZOE vehicle. This paper reflects solely the views of the authors and not necessarily the views of the company they belong to.

\section{REFERENCES}

[1] J. Snider, "Automatic steering methods for autonomous automobile path tracking," 042011.

[2] S. G. Yi, C. M. Kang, S. Lee, and C. C. Chung, "Vehicle trajectory prediction for adaptive cruise control," in 2015 IEEE Intelligent Vehicles Symposium (IV), 2015, pp. 59-64.

[3] E. Hellström, M. Ivarsson, J. Åslund, and L. Nielsen, "Look-ahead control for heavy trucks to minimize trip time and fuel consumption," Control Engineering Practice, vol. 17, pp. 245-254, 022009.

[4] V. Turri, B. Besselink, J. Mårtensson, and K. H. Johansson, "Fuelefficient heavy-duty vehicle platooning by look-ahead control," in 53rd IEEE Conference on Decision and Control, 2014, pp. 654-660.

[5] A. Alam, J. Mårtensson, and K. H. Johansson, "Look-ahead cruise control for heavy duty vehicle platooning," in 16th International IEEE Conference on Intelligent Transportation Systems (ITSC 2013), 2013, pp. $928-935$.

[6] V. Turri, B. Besselink, and K. H. Johansson, "Cooperative look-ahead control for fuel-efficient and safe heavy-duty vehicle platooning," IEEE Transactions on Control Systems Technology, vol. 25, no. 1, pp. 12-28, 2017.

[7] U. Ozguner, K. A. Unyelioglu, and C. Hatipoglu, "An analytical study of vehicle steering control," in Proceedings of International Conference on Control Applications, 1995, pp. 125-130.

[8] S. Patwardhan, Han-Shue Tan, and J. Guldner, "A general framework for automatic steering control: system analysis," in Proceedings of the 1997 American Control Conference (Cat. No.97CH36041), vol. 3, 1997, pp. 1598-1602 vol.3.

[9] J. Kosecka, R. Blasi, C. J. Taylor, and J. Malik, "Vision-based lateral control of vehicles," in Proceedings of Conference on Intelligent Transportation Systems, 1997, pp. 900-905.

[10] J. Kosecka, R. Blasi, C. J. Taylor, and J. Malik, "A comparative study of vision-based lateral control strategies for autonomous highway driving," in Proceedings. 1998 IEEE International Conference on Robotics and Automation (Cat. No.98CH36146), vol. 3, 1998, pp. 1903-1908.

[11] P. Hingwe and M. Tomizuka, "A variable look-ahead controller for lateral guidance of four wheeled vehicles," in Proceedings of the 1998 American Control Conference. ACC (IEEE Cat. No.98CH36207), vol. 1, 1998, pp. 31-35 vol.1.

[12] Chieh Chen and Han-Shue Tan, "Steering control of high speed vehicles: dynamic look ahead and yaw rate feedback," in Proceedings of the 37th IEEE Conference on Decision and Control (Cat. No.98CH36171), vol. 1, 1998, pp. 1025-1030 vol.1.
[13] M. G. Skarpetis, F. N. Koumboulis, and P. Papanikolaou, "Vehicle lateral control using a robust tracking controller based on vision look ahead system," in 2017 IEEE 21st International Conference on Intelligent Engineering Systems (INES), 2017, pp. 000213-000218.

[14] M. Park, S. Lee, and W. Han, "Development of steering control system for autonomous vehicle using geometry-based path tracking algorithm," ETRI Journal, vol. 37, 052015.

[15] F. Roselli, M. Corno, S. M. Savaresi, M. Giorelli, D. Azzolini, A. Irilli, and G. Panzani, " $\mathcal{H}_{\infty}$ control with look-ahead for lane keeping in autonomous vehicles," in 2017 IEEE Conference on Control Technology and Applications (CCTA), 2017, pp. 2220-2225.

[16] H.-S. Tan and J. Huang, "Design of a high-performance automatic steering controller for bus revenue service based on how drivers steer," Robotics, IEEE Transactions on, vol. 30, pp. 1137-1147, 102014.

[17] K. Hasegawa and E. Konaka, "Three look-ahead distance scheme for lateral control of vision-based vehicles," in 2014 Proceedings of the SICE Annual Conference (SICE), 2014, pp. 660-665.

[18] S. Lee, Y. O. Lee, Y. Son, and C. C. Chung, "Multirate active steering control for autonomous vehicle lateral maneuvering," in 2012 IEEE Intelligent Vehicles Symposium, 2012, pp. 772-777.

[19] C. M. Kang, S. Lee, and C. C. Chung, "A comparative study of lane keeping system: Dynamic and kinematic models with look-ahead distance," in 2015 IEEE Intelligent Vehicles Symposium (IV), 2015, pp. 1038-1043.

[20] S. L. C. M. Kang and C. C. Chung, "Linear parameter varying design for lateral control using kinematics of vehicle motion," in 2018 Annual American Control Conference (ACC), 2018, pp. 3239-3244.

[21] Y. Kuwata, J. Teo, S. Karaman, G. Fiore, E. Frazzoli, and J. How, "Motion planning in complex environments using closed-loop prediction," 082008.

[22] Y. Kuwata, J. Teo, G. Fiore, S. Karaman, E. Frazzoli, and J. P. How, "Real-time motion planning with applications to autonomous urban driving," IEEE Transactions on Control Systems Technology, vol. 17, no. 5, pp. 1105-1118, 2009.

[23] Y.-x. Shan, W. Yang, C. Chen, J. Zhou, L. Zheng, and B. li, "Cfpursuit: A pursuit method with a clothoid fitting and a fuzzy controller for autonomous vehicles," Int J Adv Robot Syst, 092015.

[24] L. Chen, N. Liu, Y. Shan, and L. Chen, "A robust look-ahead distance tuning strategy for the geometric path tracking controllers," in 2018 IEEE Intelligent Vehicles Symposium (IV), 2018, pp. 262-267.

[25] H. Atoui, V. Milanes, O. Sename, and J. J. Martinez, "Design And Experimental Validation of a Lateral LPV Control of Autonomous Vehicles," in Accepted to IEEE ITSC, 2020.

[26] H. Pacejka, Tire and vehicle dynamics. Elsevier, 2005.

[27] R. Rajamani, Vehicle dynamics and control. New York, USA: Springer Science \& Business Media, 2011. [Online]. Available: http://standards.sae.org/j3016201401

[28] H. Atoui, O. Sename, V. Milanes, and J. J. Martinez, "LPVBased Autonomous Vehicle Lateral Controllers: A Comparative Analysis," Apr. 2021, under review. [Online]. Available: https: //hal.archives-ouvertes.fr/hal-03197895

[29] F. Wu, "Control of linear parameter varying systems," Ph.D. dissertation, University of California at Berkeley, 1995.

[30] C. Briat, Linear Parameter-Varying and Time-Delay Systems. Analysis, Observation, Filtering Control. Springer-Verlag, 2015. 